\title{
Spatio-temporal modeling of lung images for cancer detection
}

\author{
LI SHEN $^{1}$, WEI ZHENG ${ }^{2}$, LING GAO $^{3}$, HENG HUANG $^{2}$, FILLIA MAKEDON $^{2}$ and JUSTIN PEARLMAN ${ }^{3}$ \\ ${ }^{1}$ Image and Pattern Analysis Laboratory, Department of Computer and Information Science, University of Massachusetts \\ Dartmouth, North Dartmouth, MA 02747; ${ }^{2}$ Dartmouth Experimental Visualization Laboratory, Department of Computer \\ Science, Dartmouth College, Hanover, NH 03755; ${ }^{3}$ Advanced Imaging Center, Departments of Radiology \\ and Cardiology, Dartmouth Medical School, Lebanon, NH 03756, USA
}

Received July 7, 2005; Accepted October 3, 2005

\begin{abstract}
Perfusion magnetic resonance imaging (pMRI) is an important tool in assessing tumor angiogenesis for the early detection of lung cancer. This study presents a novel integrated framework for spatio-temporal modeling of pulmonary nodules in pMRI image sequences. After localizing a nodule region in each image, we perform segmentation in the region to extract the nodule boundary, then use thin-plate spline interpolation for nodule registration along the temporal dimension. The resulting spatio-temporal model can lead to many types of nodule characterization, e.g. a time-intensity profile of a nodule region, and be used to capture important angiogenic patterns in the lung that can distinguish between cancer and benign nodules and assist in early detection.
\end{abstract}

\section{Introduction}

Lung cancer is among the most commonly occurring malignancies in the world and the leading cause of cancer death in both men and women in the U.S. Computed tomography (CT) can identify suspicious lesions, but its high false-positive rate often requires additional imaging tests within 3-6 months to confirm a positive result. To detect lung cancer at an earlier stage, an alternative and promising method is to apply a modified perfusion magnetic resonance imaging (pMRI) (1) to assess tumor angiogenesis. Angiogenesis, defined as the formation and development of new blood vessels essential for tumor growth and the eventual spread of cancer cells to secondary locations throughout the body, is one of the most promising areas of investigation in cancer research.

One key issue in the analysis of pMRI lung image sequences is to effectively characterize angiogenic patterns

Correspondence to: Dr Li Shen, Department of Computer and Information Science, University of Massachusetts Dartmouth, 285 Old Westport Road, North Dartmouth, MA 02747, USA

E-mail: 1shen@umassd.edu

Key words: perfusion magnetic resonance imaging, pulmonary nodule, segmentation, registration, time-intensity profile of pulmonary nodules. To achieve this goal, we present a novel and automated spatio-temporal modeling technique for pulmonary nodules in pMRI sequences, and demonstrate our approach by creating a time-intensity profile for any userinterested nodule region. Many techniques of nodule detection (2) and segmentation (3) have been developed based on chest radiographs or CT images. New challenges arising in our analysis include: i) as a unique imaging modality, pMRI requires new processing techniques for detection and segmentation of lung nodules; and ii) to compensate for respiratory and cardiovascular motion in pMRI sequences, registration needs to be done to ensure that corresponding nodule occurrences in different time frames are comparable.

\section{Materials and methods}

Prior written consent was obtained as required by the Hospital Research Board. Surface EKG electrodes were used for cardiac cycle timing during the MR imaging procedure. MR imaging was performed using an eight-channel phased-array coil of a 1.5-T whole-body GE dual gradient Excite magnet. After scout images of the chest, a bolus fast gradient-echo train imaging (FGRET) was performed at the rate of one image per heartbeat with flip angle adjusted to null the target lesion on the fourth and subsequent frames prior to arrival of the contrast agent $\left(\mathrm{TR} / \mathrm{TE} / \mathrm{flip}\right.$ angle $=6.06 \mathrm{~ms} / 1.108 \mathrm{~ms} / 75^{\circ}$, slice thickness $=20 \mathrm{~mm}$, views per segment $=4$, matrix $=256 \times 256$, field of view $=440 \times 440 \mathrm{~mm}$, and EKG-triggered $\mathrm{RR}=1$ ). Likewise, static inversion recovery images were adjusted to null the target lesion, typically using TI $=250-300 \mathrm{msec}$. While imaging by FGRET, $20 \mathrm{ml}$ of Gd-DTPA (Magnevist; Berlex Laboratories) was injected into the venous line as a time series of 84 images were obtained with one frame per heartbeat observing signal changes relating to arrival and washout of the contrast agent. Subsequently, at 5,10 and 15 min after injection, delayed enhancement static inversion recovery images were obtained to assess agent retention, which occurs in damaged cells. In this study, we analyze the time series of images obtained during the first-pass of the bolus of contrast agent to demonstrate our spatio-temporal modeling technique. Fig. 1 shows four examples from this series of 84 images.

Our overall strategy is to divide the problem into several relatively simple subproblems. Generally speaking, image registration is a difficult task (4). However, if the relevant 
Slice 1

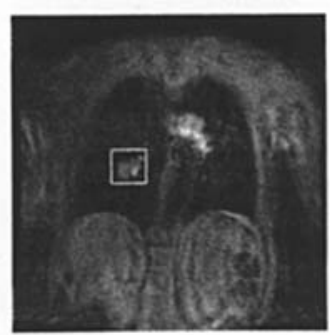

Slice 4

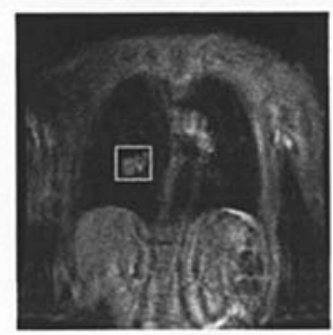

Slice 16

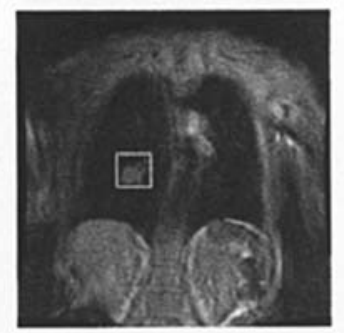

Slice 64

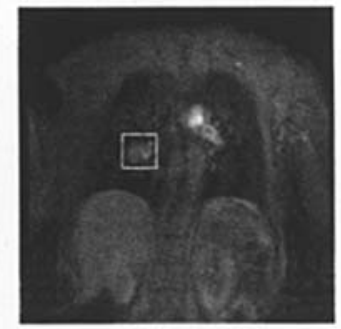

Figure 1. Nodule region identification. In each slice, the rectangular box indicates the identified nodule region.
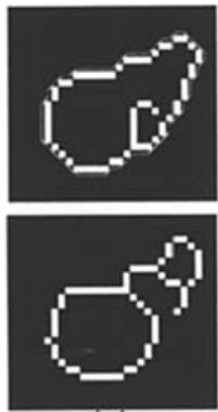

(a)
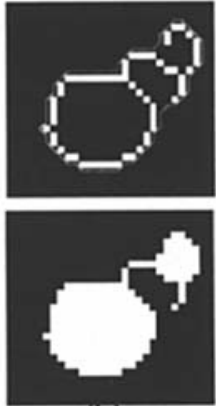

(b)
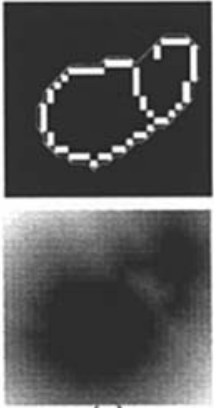

(c)
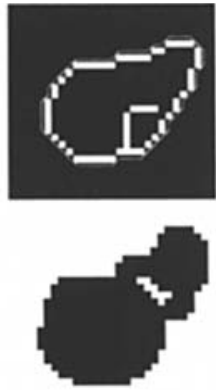

(d)
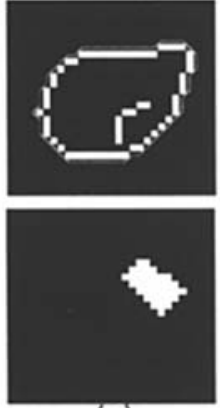

(e)
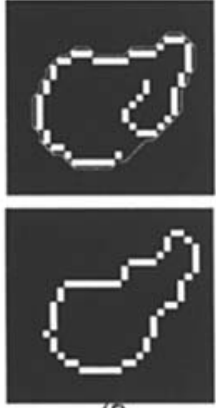

$(\mathrm{f})$

Figure 2. Nodule boundary segmentation. The first row shows sample edge detection results (white pixels) and segmentation results (contours). The second row shows a sample procedure of closing broken edges: (a) intital edges, (b) bridge unconnected pixels and fill image region, (c) distance transformation map, (d) threshold distance transformation map and find connected components, (e) remove the background component and slightly dilate the rest of the components, and (f) extract the boundary of (b) and (e).

nodule region of our focus is localized, registration of this sub-image between different time frames becomes a much easier task. More conveniently, the nodule boundary from this sub-image can be extracted, and the segmentation results can be used to complete nodule registration. Thus, our framework includes 3 steps: i) nodule identification in each slice; ii) nodule segmentation in the relevant region; and iii) nodule registration using segmentation results. The following subsections describe these steps in more detail.

Identification. To identify a local nodule region in each image slice, a simple method is employed. In one slice, we manually define a rectangular region A to enclose the nodule of interest. In other words, $\mathrm{A}$ is an $\mathrm{m} \times \mathrm{n}$ sub-image that contains the nodule. In our experiments, we define A as the first slice, which has a size of $26 \times 28$.

In each of the other slices, we search for a region $B$ of the same size that maximizes the correlation coefficient between $A$ and $B$. The two-dimensional correlation coefficient between two matrices $A$ and $B$ of the same size is given by the formula:

$r=\left(\sum_{m} \sum_{n}\left(A_{m n}-\bar{A}\right)\left(B_{m m}-\bar{B}\right)\right) / \sqrt{\left(\sum_{m} \sum_{n}\left(A_{m n}-\bar{A}\right)^{2}\right)\left(\sum_{m} \sum_{n}\left(B_{m n}-\bar{B}\right)^{2}\right)}$

where $\bar{X}$ denotes the mean of elements of matrix X.

In our implementation, after initializing $B$ to have the same location as $A$, we move $B$ in the neighborhood area, calculate the correlation coefficient for each case, then select the best one. This approach is simple and works perfectly on our data. The nodule region is correctly identified in all of the slices. Fig. 1 shows four sample image slices with identified nodule regions.
Segmentation. Nodule segmentation is performed on each sub-image identified in the previous step, which reduces the complexity of the segmentation task. Fig. 4 shows sample nodule sub-images, and the nodule appearance in these subimages is relatively clear. Thus, a few simple image processing techniques can be combined to complete the segmentation task.

A segmentation procedure was designed as follows: i) apply a filter to reduce the contribution of noise, e.g. $3 \times 3$ average, median, or median-average filter (average was used in our experiments); ii) apply Canny edge detection to extract object edges; and iii) if necessary, close the object edges to form a simply-connected object, using an approach described in the next paragraph. The Canny method used in the second step finds edges by looking for local maxima of the gradient of the image. The gradient is calculated using the derivative of a Gaussian filter. The method uses two thresholds to detect strong and weak edges, and includes the weak edges in the output only if they are connected to strong edges. This method is known to many as the optimal edge detector.

The first row of Fig. 2 shows six sample results after performing the first two steps of the segmentation procedure. Some of these results (Fig 2a, d and e) form a closed nodule region, while others (Fig. 2b, c and f) do not. The third step was designed to derive a closed nodule boundary: a) start from the edge detection result; b) bridge previously unconnected pixels (works only for small gaps), then fill the image regions; c) calculate the distance transformation map; d) threshold the transformation map ( $t=1.5$ in our experiments) and find its connected components; (e) remove the background component and slightly dilate the remaining 

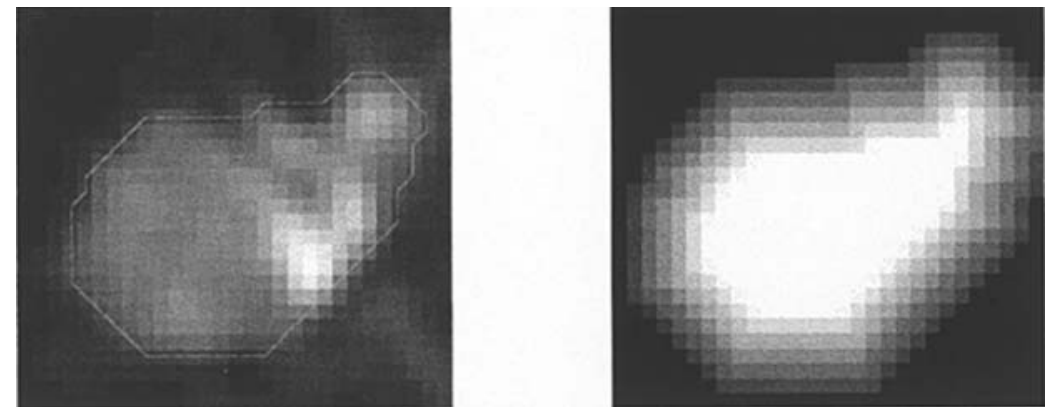

Figure 3. Mean nodule image (left), mean nodule boundary (contour) and weighting mask (right).

Slice 1

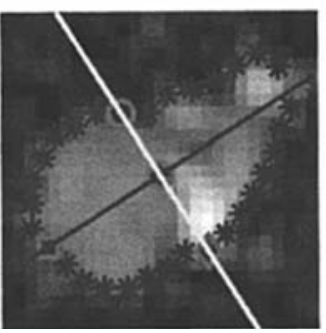

Slice 4

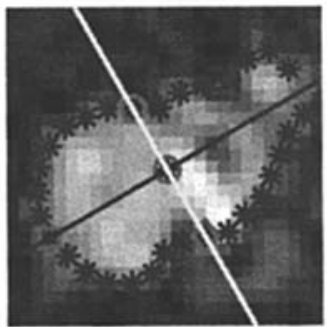

Slice 16

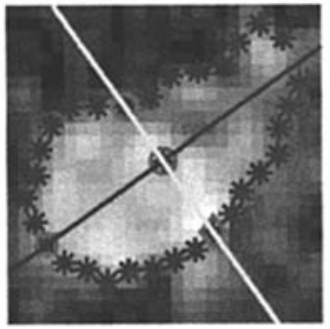

Slice 64

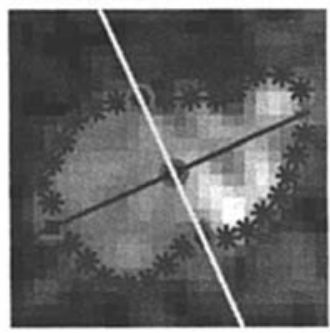

Figure 4. Principal axes and landmarks (consistent across subjects). Image intensity is scaled for better visualization

components (a disk with radius $=3$ was used as the morphological structuring element in our experiments); and f) add the results of b) and e) and extract its boundary. The second row of Fig. 2 shows a sample run of the procedure.

The above segmentation procedure works for most of the nodule sub-images. For some noisy cases, however, it fails to create a reasonable nodule shape. To overcome this problem, we introduced a weighting mask to capture a general nodule shape, then applied it to each image to impose some structural constraint on the segmentation results.

The following approach was used to calculate the weighting mask: i) scale the intensity values to the range from 0 (black) to 1 (white) for each sub-image, and calculate the mean image; ii) segment the mean using the procedure described above; iii) calculate the signed distance transformation map of the mean boundary; and iv) threshold the transformation map so the most positive and negative values become $t$ and $-t$ respectively ( $t=3$ in our experiments).

Fig. 3 shows the mean nodule boundary and weighting mask. Since the weighting mask captures a general nodule shape, it was multiplied with each nodule image on a pixelby-pixel basis, then the segmentation described above was performed. Clearly, this approach imposes structural constraint on each individual nodule so its shape will not deviate from a typical nodule shape. Using this strategy, satisfactory results were obtained for all images. Fig. 4 shows some examples (see stars).

Registration. After segmentation, the spatial characteristics of a nodule are available at each time point. To build a spatio-temporal nodule model, registration along the temporal dimension needs to be completed. In other words, for each pixel inside the nodule in the first slice, its corresponding pixels need to be identified in all subsequent slices. A landmark-based registration approach was developed to include the automatic identification of landmarks in each slice and thin-pline spline interpolation for image matching (5).

A landmark is a point of correspondence on each nodule occurrence that matches between time frames. We developed a procedure to automatically extract a set of landmarks in each slice by performing principal component analysis (PCA) (6) on each nodule represented by a binary image to find its principal axes. These principal axes intersect the nodule boundary at four points, which become our base landmarks. More landmarks are then extracted by finding the midpoint of the contour between two neighboring landmarks and defining it as a new landmark. Besides these boundary landmarks, the nodule centroid was included as an additional landmark. In our experiments, we used 32 boundary landmarks and 1 centroid landmark. Fig. 4 shows the PCA axes and sample landmarks, which are labeled and consistent across subjects.

Landmarks define the correspondence at the nodule boundary and centroid. To create correspondence for the interior part, thin-plate spline interpolation was employed (5). A thin-plate spline $f(x, y)$ is a smooth function that interpolates a surface, which is fixed at the landmark points $P_{i}$ at a specific height $h_{i}$. This surface can be imagined as a thin metal plate that will take on a shape in which it is least bent, i.e. it minimizes the bending energy of $f(x, y)$ :

$$
\iint_{R^{2}}\left(\frac{\partial^{2} f}{\partial x^{2}}\right)^{2}+\left(\frac{\partial^{2} f}{\partial x \partial y}\right)^{2}+\left(\frac{\partial^{2} f}{\partial y^{2}}\right)^{2} d x d y
$$

In order to map one image to another using landmark data, two of these spline surfaces are computed, one $\left(f_{x}\right)$ in which the heights $\left(h_{i}\right)$ are loaded with the x-coordinate of the landmarks $\left(P_{i}\right)$ in the second image, and another $\left(f_{y}\right)$ for the $y$-coordinate. The first of these functions supplies the interpolated $\mathrm{x}$-coordinate of the map we seek, and the second 
Slice 1

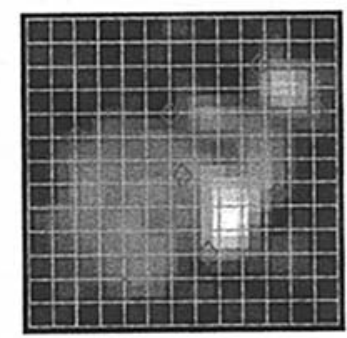

Slice 4

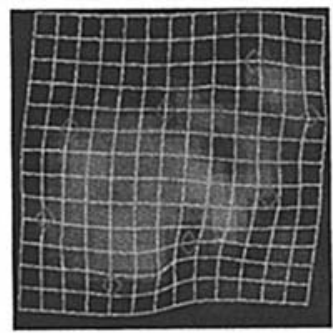

Slice 16

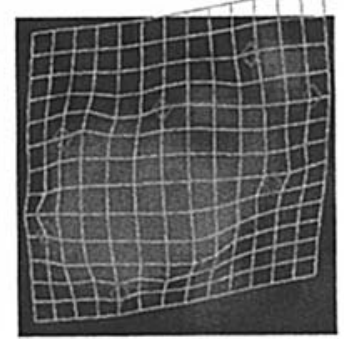

Slice 64

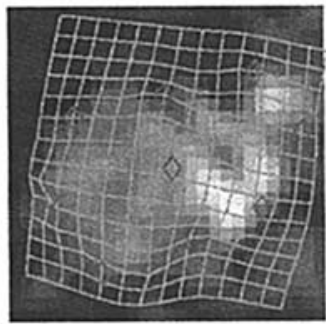

Figure 5. Thin plate interpolation. The registration results are visualized by meshes, and image intensity is not scaled.

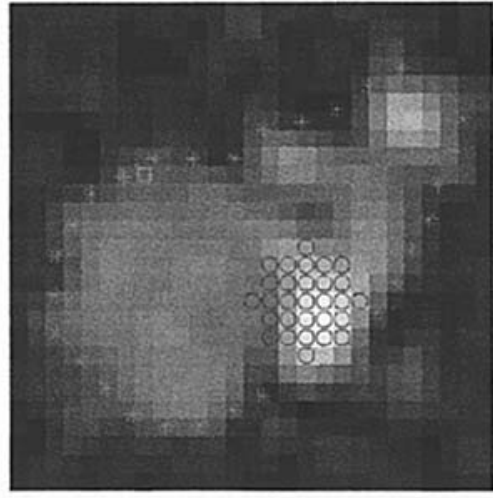

(a)



(b)

Figure 6. (a) Three ROIs. A single pixel (square), the whole nodule (bounded by stars), and a region (circles). (b) Time intensity profiles of these ROIs.

supplies the interpolated y-coordinate. The resulting map $\left[f_{x}(P), f_{y}(P)\right]$ is now a deformation of one image plane onto the other that maps landmarks onto their homologues and has the minimum bending energy of any such interpolant.

Fig. 5 shows sample results of applying thin-plate spline interpolation to our data. Clearly, given any nodule pixel in the first slice, the corresponding pixels in all other slices can then be found.

\section{Results}

We demonstrated our spatio-temporal modeling technique using a pMRI image sequence described above, which comprised 84 lung perfusion, short-axis, MR images obtained from a freely breathing patient. Identification, segmentation, and registration algorithms were applied to these images, and Figs. 1, 4 and 5 shows sample results at different stages. After these steps, a spatio-temporal model was established for the nodule of interest.

Using this model, a time-intensity profile can be created for a region of interest (ROI) in the nodule, which can be specified in the first slice. According to our model, the corresponding ROI in all other slices can be easily extracted. Thus, a time-intensity profile for the ROI could be represented as a curve showing the change in mean intensity (or intensity variance) over time.

This profile in relation to an upstream arterial profile defines the point-spread function for blood delivery to the observed loci, which is largely determined by the convolution effects from small pathways (angiogenesis). In particular, if the blood delivery pattern is consistent with increased microvascular development, this implicates tumor angiogenesis, and timing with respect to pulmonary vein enhancement can help distinguish primary lung cancers from metastases.

Fig. 6 shows several ROIs and their profiles by plotting the mean intensity versus time. These time-intensity profiles can capture different types of angiogenic patterns of the pulmonary nodule and have the potential to distinguish cancer nodules from benign ones.

\section{Discussion}

We presented an integrated framework for spatio-temporal modeling of pulmonary nodules in pMRI image sequences, and demonstrated its application in creating time-intensity profiles for any nodule regions of interest. This spatiotemporal model can also lead to many other types of nodule characterization, e.g. volume/shape change over time. One future direction is to use this model to extract important angiogenic patterns in the lung, which may effectively discriminate between cancer and benign nodules and help to accurately detect lung cancer in its early stages.

\section{Acknowledgements}

This work was supported by the Flight Attendant Medical Research Institute. 


\section{References}

1. Fink C, Puderbach M, Bock M, et al: Regional lung perfusion: assessment with partially parallel three-dimensional MR imaging. Radiology 231: 175-184, 2004.

2. Lee Y, Hara T, Fujita H, et al: Automated detection of pulmonary nodules in helical CT images based on an improved templatematching technique. IEEE Trans Med Imaging 20: 595-604, 2001 .

3. $\mathrm{Xu} \mathrm{N}$, Ahuja N and Bansal R: Automated lung nodule segmentation using dynamic programming and EM-based classification. Medical Imaging 2002: Image Processing. Sonka M and Fitzpatrick JM (eds). Proceedings of SPIE 4684: 666-676, 2002.
4. Maintz J and Viergever M: A survey of medical image registration. Med Image Anal 2: 1-36, 1998.

5. Bookstein F: Principal warps: thin-plate splines and the decomposition of deformations. IEEE Trans Pattern Anal Machine Intelligence 11: 567-585, 1989

6. Duda RO, Hart PE and Stork DG: Pattern Classification. 2nd edition. Wiley, New York, NY, 2000. 